\title{
Neuromuskuläre Übertragungsstörungen
}

Jörn Peter Sieb

\begin{abstract}
Entsprechend der Heterogenität der zugrundeliegenden Störungen ist das klinische Bild uneinheitlich. Nur teilweise ist eine myasthene Schwäche offensichtlich, d. h. die bei muskulärer Belastung sich akzentuierende Muskelschwäche. Der Neurophysiologie kommt die diagnostische Schlüsselrolle bei der Identifikation von Störungen der neuromuskulären Signalübertragung zu. Mit den eingesetzten Methoden der Serienstimulation und dem Einzelfaser-EMG ist es möglich, eine Störung der neuromuskulären Signalübertragung zu dokumentieren und damit den Impuls für eine zielgerichtete weitere Diagnostik zu geben mit dem Ziel der eindeutigen Ursachenidentifikation.
\end{abstract}

\section{Definition}

\section{ÜBERSICHT/SYSTEMATIK}

Die synaptische Signalübertragung an der motorischen Endplatte zwischen Nerv und Muskel kann in vielfältiger Weise gestört sein. Nach der Verursachungsart werden unterschieden:

- angeboren (=kongenitale Myasthenie-Syndrome)

- immunologisch (z. B. Lambert-Eaton-Syndrom, Myasthenia gravis)

- toxisch (z. B. Botulismus)

Weiterhin werden die Störungen nach ihrer Lokalisation eingeteilt in

- präsynaptisch (z. B. Lambert-Eaton-Syndrom)

- synaptisch (z. B. angeborene Acetylcholinesterase-Defizienz)

- postsynaptisch (z. B. Myasthenia gravis)

\section{Allgemeines Vorgehen -} von der Diagnose zur Therapie Allgemeines Vorgehen

Bei jeder diagnostisch unklaren Muskelschwäche sollte an die Möglichkeit einer Störung der neuromuskulären Signalübertragung gedacht werden. Erster Schritt der Diagnostik ist die niederfrequente Serienstimulation gegebenenfalls mit Provokationsmethoden, wie eine Untersuchung in der posttetanischen Erschöpfungsphase. Ergänzend erfolgen elektrophysiologisch gegebenenfalls weitere Untersuchungsverfahren, wie ein Einzelfaser-EMG ( $\mathbf{A} \mathbf{b} \mathbf{b} \mathbf{b} \mathbf{1}$ ). Je nach klinischer Situation sind dann weitere serologische, bildgebende und auch molekulargenetische Untersuchungen erforderlich.

\section{Spezielle Erkrankungen}

\section{Myasthenia gravis}

\section{DEFINITION}

Die Bezeichnung „Myasthenia gravis“ (MG) umfasst eine heterogene Gruppe von Autoimmunerkrankungen, deren gemeinsames Merkmal eine autoimmun bedingte, postsynaptische Störung der Signalübertragung an der motorischen Endplatte ist [4].

\section{Systematik}

Die Unterteilung erfolgt nach:

- Verlaufstyp:

- okulär (bei zirka 20\% aller Patienten)

- oropharyngeal bzw. generalisiert

- Erkrankungsbeginn:

- neonatal (passager, durch den transplazentaren Transfer mütterlicher Antikörper)

- vor Beginn der Pubertät (insbesondere bei Asiaten)

- bei jüngeren Erwachsenen (in der Literatur Altersgrenze uneinheitlich: meist Erkrankungsbeginn vor dem 50. Geburtstag)

- bei älteren Erwachsenen

- Antikörperspezifität:

- anti-AChR (Acetylcholinrezeptor)

- anti-MuSK (Muskel-spezifische Rezeptor-Tyrosinkinase) 


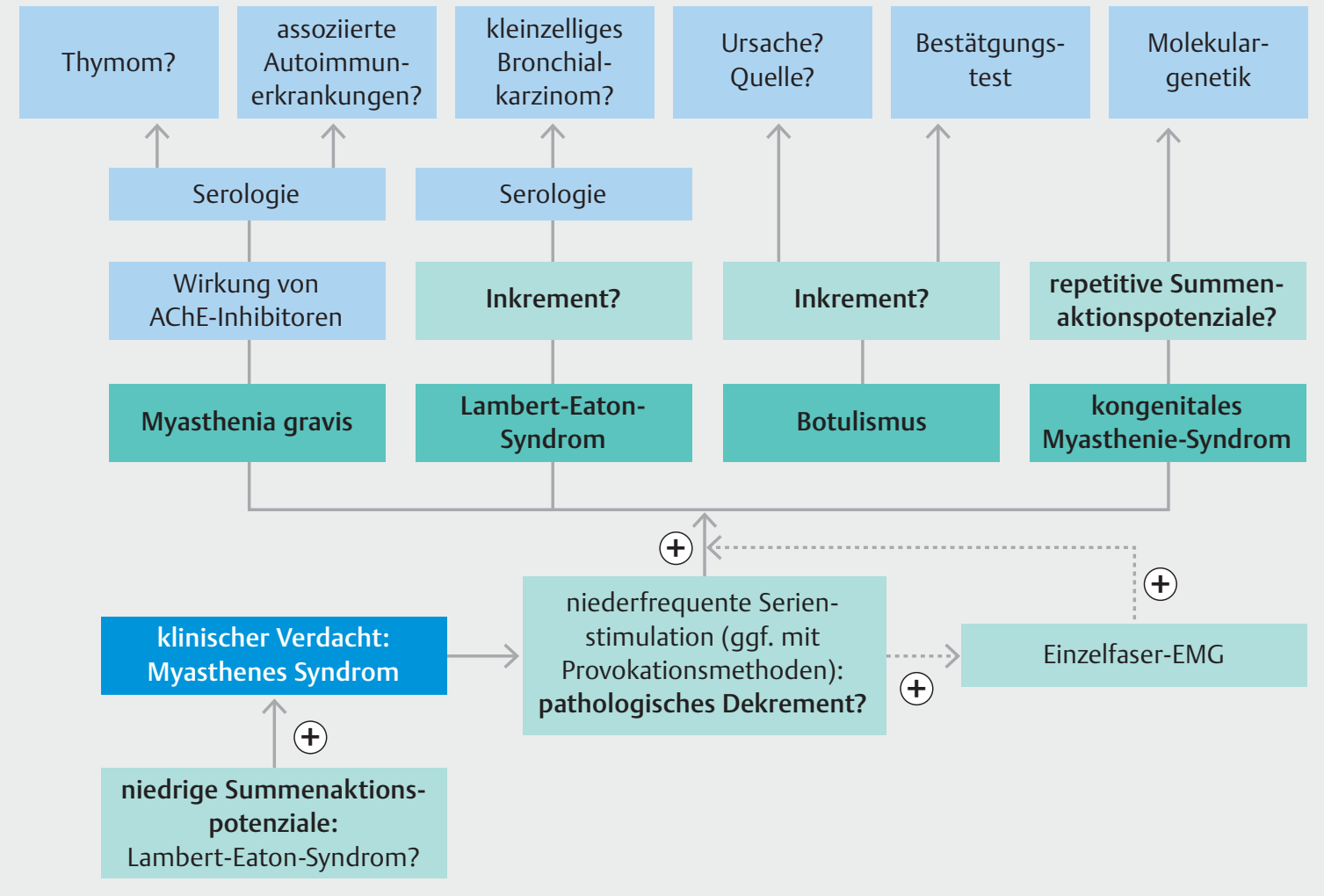

Abb. 1 Neuromuskuläre Übertragungsstörungen: allgemeines Vorgehen.

- anti-LRP4 (low-density lipoprotein receptorrelated protein 4)

- weitere Antikörper mit noch unklarer klinischer Relevanz (wie z. B. anti-Agrin)

- seronegativ: kein Nachweis krankheitsspezifischer Antikörper

- Thymushistologie:

- normal bzw. atrophisch

- Thymitis

- Thymom (paraneoplastische MG)

\section{Diagnostik}

\section{Die Diagnostik erfolgt}

- neurophysiologisch: niederfrequente Serienstimulation, Einzelfaser-EMG. Hinweis: Bei der anti-MuSKpositiven Myasthenie kann das Einzelfaser-EMG falsch negative Resultate liefern. Weiterhin kann bei dieser Myasthenie-Form ein Wiederanstieg der Amplitude nach dem 5. Summenaktionspotenzial während einer niederfrequenten Serienstimulation fehlen, wie dies auch beim Lambert-Eaton-Syndrom beobachtet wird [3]. Bei dieser Myasthenie-Form zeigt sich gelegentlich ein myopathisches EMGMuster [2].

- serologisch: Nachweis krankheitsspezifischer Antikörper, was teilweise aber nicht gelingt.
- pharmakologisch: Besserung eines Zielsymptoms durch die Gabe eines Acetylcholinesterase-Inhibitors. Diese Testung ist unspezifisch und es gibt bei der anti-MuSK-positiven Myasthenie falsch negative Ergebnisse.

\section{FALLEN/PROBLEME}

Die Sensitivität der Untersuchungsverfahren ist nicht hoch. Keineswegs ist es möglich, mit einem dieser Untersuchungsverfahren das Vorliegen einer Myasthenie auszuschließen.

\section{Zusätzliche Diagnostik}

- Liegen ausschließlich Hirnnervensymptome vor, dann ist ein zerebrales MRT sinnvoll.

- Thoraxbildgebung CT/MRT (Thymom?), ggf. ergänzt durch nuklearmedizinische Untersuchungen

- Suche nach assoziierten Autoimmunerkrankungen. Die Kombination mit einem Lambert-Eaton-Syndrom (Overlap-Syndrom) sollte nur angenommen werden, wenn beide Erkrankungen elektrophysiologisch und serologisch eindeutig zu diagnostizieren sind. 


\section{Therapie}

Aus 4 Behandlungsoptionen wird ein individueller Therapieplan erstellt angepasst an die jeweilige Krankheitssituation [4]:

- Verbesserung der neuromuskulären Signalübertragung durch Acetylcholinesterase-Inhibitoren, wie Pyridostigminbromid

- Behandlung bei einer drohenden respiratorischen Insuffizienz (myasthene Krise): Plasmapherese, Immunadsorption, intravenöse Immunglobuline

- Immunsuppression (Prednisolon, Azathioprin, Mycophenolat, Rituximab, Eculizumab u. a.)

- Thymektomie

\section{Lambert-Eaton-Syndrom}

\section{DEFINITION}

Dies ist eine präsynaptische Störung der neuromuskulären Signalübertragung. Ursache ist eine Immunantwort gegen präsynaptische Kalziumkanäle vom P/Q-Typ. Diese Kalziumkanäle öffnen sich während der Depolarisation bei einem nervalen Aktionspotenzial, so dass Kalziumionen in die Nervenendigung einströmen. Kalzium ist erforderlich für die Acetylcholin-Ausschüttung. Entsprechend ist beim Lambert-Eaton-Syndrom die Acetylcholin-Ausschüttung pro Aktionspotenzial reduziert. Es wird eine idiopathische und eine paraneoplastische Form unterschieden [5]. Die Störung betrifft nicht nur die motorische Endplatte, sondern auch cholinerge Synapsen des autonomen Nervensystems.

\section{Symptome}

- Beginn typischerweise mit einer Hüftgürtelschwäche

- Ca. 50\% der Fälle treten paraneoplastisch im Rahmen eines kleinzelligen Bronchialkarzinoms auf.

- Kombination mit autonomen Symptomen (u. a. störend trockener Mund, Hypohidrose, Erektionsstörung)

\section{Grundprogramm}

- Darstellung der Lambert-Trias ( $>$ Abb. 2):

- niedrige Summenaktionspotenziale in der motorischen Neurografie; an den Handmuskeln häufig Peak-to-peak-Amplitude unter $2 \mathrm{mV}$

- pathologisches Dekrement (>9\%) in der niederfrequenten Serienstimulation, wobei sich typischerweise kein Wiederanstieg der Amplituden nach dem 5. Summenaktionspotenzial zeigt [3]

- Inkrement. Durch eine maximale Willkürinnervation über 10 Sekunden nimmt die Amplitude der Summenaktionspotenziale deutlich zu. Diese

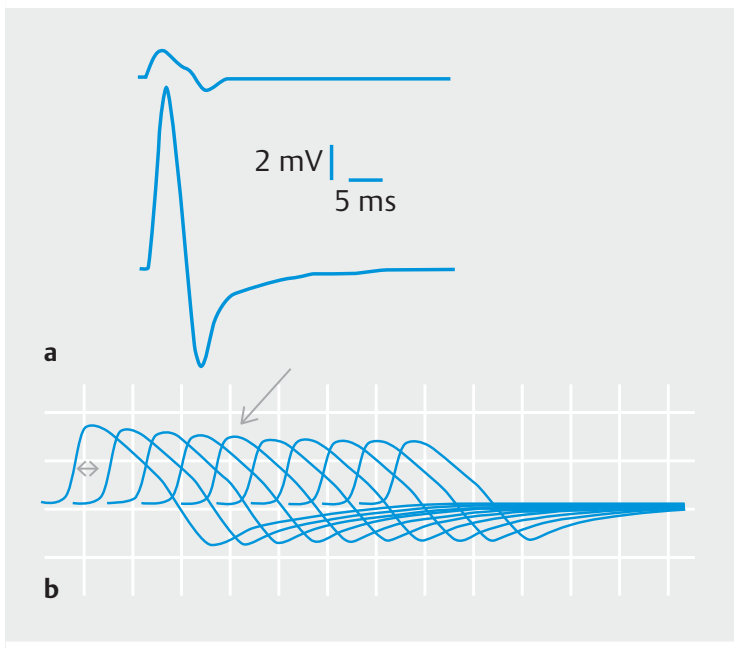

- Abb. 2 Elektrophysiologische Lambert-Trias bei einem nicht-paraneoplastischen Lambert-Eaton-Syndrom. (a) Niedrige Amplituden der motorischen Summenaktionspotenziale, Inkrement durch maximale Willküraktivität über 10 Sekunden. Dargestellt wird das motorische Summenaktionspotenzial des M. abductor digiti quinti, supramaximale Stimulation des N. ulnaris am Handgelenk, Ableitung mit Oberflächenelektroden. Die Ausgangsamplitude beträgt 2,2 mV (oben). Nach der Muskelanspannung Anstieg der Amplitude auf 15,4 mV, was einem Inkrement von $700 \%$ entspricht (unten). (b) Pathologisches Dekrement der Summenaktionspotenziale des M. trapezius von $14 \%$ (Vergleich 1./5. Summenaktionspotenzial), supramaximale Stimulation des N. accessorius, Ableitung mit Oberflächenelektroden). Wie für das Lambert-EatonSyndrom typisch, kommt es hier zu keinem Wiederanstieg der Amplituden nach dem 5. Potenzial (Pfeil).

Amplitudenzunahme, das Inkrement, wird durch einen vermehrten Kalziumeinstrom während der Willküraktivität erklärt. Bei einer längeren Muskelanspannung geht das Inkrement wieder zurück. Beim Lambert-Eaton-Syndrom ohne Nachweis von Antikörpern gegen Kalziumkanäle vom P/Q-Typ sind die elektrophysiologischen Auffälligkeiten oft geringer ausgeprägt, so dass bei seronegativen Patienten ein Inkrement von $60 \%$ als diagnostisch beweisend gelten sollte. Ein Inkrement kann auch über eine hochfrequente, jedoch sehr schmerzhafte Serienstimulation mit Stimulationsfrequenzen von über $20 \mathrm{~Hz}$ dargestellt werden.

DIFFERENZIALDIAGNOSTISCHER HINWEIS Bei auffällig niedrigen Amplituden der Summenaktionspotenziale in der motorischen Neurografie sollte man an die Möglichkeit eines LambertEaton-Syndroms denken. 


\section{Zusätzliche Diagnostik}

- serologische Diagnostik (Autoantikörper gegen Kalziumkanäle vom P/Q-Typ, die bei zirka 10\% der Fälle nicht nachweisbar sind)

- intensive Suche nach einem kleinzelligen Bronchialkarzinom gerade bei älteren Rauchern

\section{Therapie}

- Besserung der neuromuskulären Signalübertragung mit dem reversiblen Kaliumkanal-Blocker Amifampridin

- Immunsuppression

- onkologische Therapie bei paraneoplastischen Fällen

\section{Botulismus}

Der lebensbedrohliche Botulismus ist inzwischen in Deutschland eine Rarität mit zumeist weniger als 10 erfassten Fällen jährlich.

\section{DEFINITION}

- Störung der synaptischen Transmission an der motorischen Endplatte und an cholinergen Synapsen des Parasympathikus.

- Wird beim Menschen durch die von Clostridium botulinum produzierten Exotoxine hervorgerufen, deren Wirkmechanismus genau aufgeklärt ist. Sie wirken als spezifische Endoproteasen von Membranproteinen, die am „Andocken“ der Transmittervesikel an die präsynaptische Membran beteiligt sind. Dies hemmt die Transmitterausschüttung.

\section{Symptome}

- bei Erwachsenen Beginn mit gastrointestinalen Symptomen und rasch zunehmenden bulbären Zeichen, dann symmetrisch absteigende, schlaffe Tetraparese und autonome Dysfunktion (anticholinerger Effekt)

\section{WICHTIGE DIFFERENZIALDIAGNOSEN}

- Myasthenia gravis: zumeist weniger foudroyant verlaufend, keine autonome Störung

- Guillain-Barré-Syndrom: sensible Mitbeteiligung, Paresen von distal aufsteigend

\section{Grundprogramm}

Die neurophysiologischen Veränderungen sind denjenigen beim Lambert-Eaton-Syndrom vergleichbar.
- In der motorischen Neurografie Summenaktionspotenziale niedriger Amplitude mit normalen distalmotorischen Latenzen und Nervenleitgeschwindigkeiten. Normale sensible Neurografie.

- pathologisches Dekrement in der niederfrequenten Serienstimulation $(2-3 \mathrm{~Hz})$ möglich

- Inkrement durch maximale Willkürinnervation über 10 s oder durch hochfrequente Serienstimulation ( $30-50 \mathrm{~Hz}$ ) (häufig nur wenig ausgeprägt und kann bei ausgeprägten Fällen sogar fehlen)

- elektromyografisch häufig Entwicklung von pathologischer Spontanaktivität als Zeichen der funktionellen Denervierung und eines scheinbar myopathischen Musters in der Einzelpotenzialanalyse

- konfirmatorische Tests (z. B. der nur wenig sensitive Maus-Inokulationstest zum Toxinnachweis aus Stuhl, Serum, Mageninhalt bzw. asservierter Nahrung)

\section{Therapie}

- intensivmedizinische Überwachung

- bei Wundbotulismus: Wunddébridement, Antibiose

- in den ersten Stunden Magenspülung, Einläufe, properistaltische Behandlung, um kontaminierte Nahrungsreste zu entfernen

- Antitoxin ist nur innerhalb der ersten 24 Stunden wirksam.

- symptomatisch Acetylcholinesterase-Inhibitoren

\section{FALLEN/PROBLEME}

- Botulismus wird häufig (zu) spät diagnostiziert.

- Schon beim Verdacht besteht Meldepflicht.

\section{Kongenitale Myasthenie-Syndrome}

\section{DEFINITION}

Diesen angeborenen Erkrankungen liegt ein breites Spektrum von Störungen der neuromuskulären Signalübertragung zugrunde [1]. Überwiegend finden sich postsynaptische Defekte. Betroffen sind z. B. die Gene für die 5 Untereinheiten des nikotinergen Acetylcholinrezeptors oder Gene für Proteine (z. B. Rapsyn, Dok-7), die für die Ausbildung der Endplattenstruktur erforderlich sind.

\section{Symptome}

Das klinische Bild ist uneinheitlich. Bei jeder unklaren Muskelschwäche sollte auch bei Erwachsenen die Möglichkeit eines kongenitalen Myasthenie-Syndroms erwogen werden. 


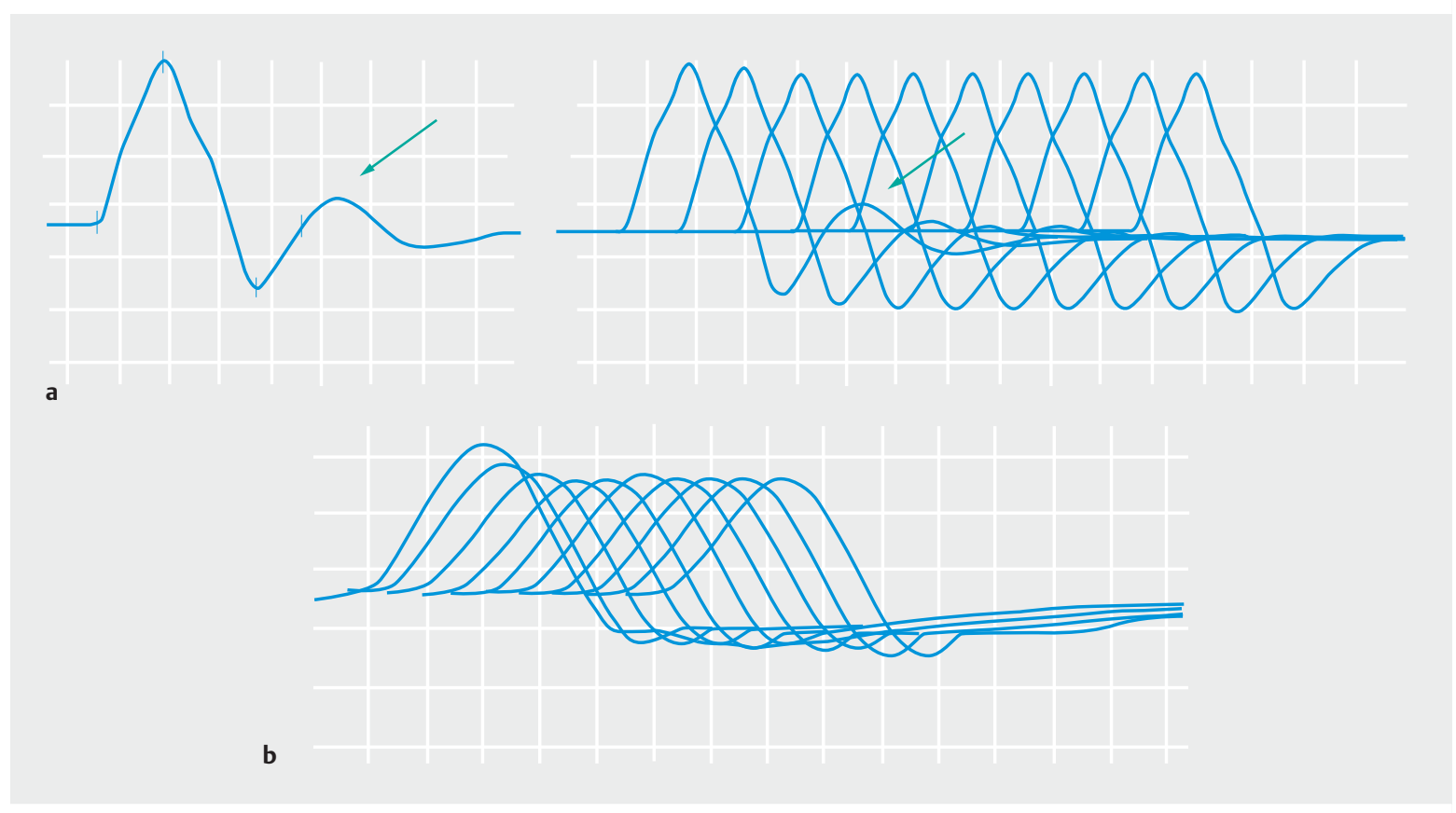

Abb. 3 Elektrophysiologische Befunde beim Slow-Channel-Syndrom. Bei diesem kongenitalen Myasthenie-Syndrom ist die Offenzeit des Acetylcholinrezeptor-Ionenkanals verlängert („gain of function“). Dies führt zu einem vermehrten Kalziumeinstrom während der Depolarisation am Beginn eines muskulären Aktionspotenzials, was wiederum eine zunehmende Degeneration des postsynaptischen Faltenapparats bedingt. Beim Slow-Channel-Syndrom zeigt sich neben einer allmählich progredienten Gliedergürtelschwäche typischerweise eine besondere ausgeprägte Schwäche der Hand- und Fingerextensoren. Dargestellt werden die bei einem 51-jährigen Mann erhobenen Befunde. Nach dem 30. Lebensjahr entwickelte sich eine Gliedergürtelschwäche mit einer inzwischen deutlich eingeschränkten Gehstrecke. (a) Es erfolgte eine supramaximale Serienstimulation mit $3 \mathrm{~Hz}$ des N. ulnaris und des N. accessorius rechts. Abgeleitet wurde mit Oberflächenelektroden vom Hypothenar bzw. vom M. trapezius. Am Hypothenar fand sich kein pathologisches Amplitudendekrement, aber repetitive Summenaktionspotenziale (Pfeile), deren Amplitude während der Reizserie rasch abnahm. Links ist das erste motorische Summenaktionspotenzial der Reizserie dargestellt. (b) Dagegen zeigte sich am M. trapezius ein ausgeprägtes Amplitudendekrement von 25\% (Vergleich 1. mit dem 5. Summenpotenzial, normal $<10 \%$ ), aber keine repetitiven Summenaktionspotenziale.

\section{FALLEN/PROBLEME}

Vielfach werden vermutlich diese Erkrankungen als seronegative Myasthenie oder Myopathie verkannt.

\section{Grundprogramm}

- Erster diagnostischer Schritt ist die elektrophysiologische Dokumentation einer Störung der neuromuskulären Signalübertragung. Teilweise ist ein pathologisches Dekrement erst durch Provokation, wie eine prolongierte $10 \mathrm{~Hz}$-Serienstimulation oder eine forcierte Muskelanspannung über Minuten, nachweisbar. In der motorischen Neurografie können sich bei der Acetylcholinesterase-Defizienz und dem Slow-Channel-Syndrom als elektrophysiologisches Charakteristikum repetitive Muskelsummenpotenziale zeigen ( $\triangleright$ Abb. $\mathbf{3}$ ).

- Der serologische Nachweis eines Immunprozesses z. B. mit Vorliegen von Anti-AcetylcholinrezeptorAntikörpern schließt das Vorliegen eines kongenitalen Myasthenie-Syndroms praktisch aus.
- Muskelhistologisch zeigen sich zumeist nur unspezifische Veränderungen. Tubuläre Aggregate als an sich unspezifische muskelhistologische Auffälligkeit weisen auf Mutationen in den Genen für die Enzyme GFPT1 bzw. DPAGT1 als Ursache von Gliedergürtelmyasthenien hin.

- Eine pharmakologische Testung mit Gabe von Acetylcholinesterase-Inhibitoren, wie Edrophoniumchlorid, ist diagnostisch nicht hilfreich. Nur ein Teil dieser Syndrome zeigt entsprechend dem zugrundeliegenden Defekt eine Zunahme der Muskelkraft durch eine Hemmung der Acetylcholinesterase-Inhibitors Edrophoniumchlorid.

- Molekulargenetisch wird kommerziell eine PanelDiagnostik angeboten. Nicht allzu selten gelingt jedoch molekulargenetisch die Identifikation der zugrundeliegenden Mutation nicht.

\section{Therapie}

Einige kongenitale Myasthenie-Syndrome können gezielt medikamentös beeinflusst werden. Dazu sei auf die spezielle Literatur zu diesen seltenen Krankheitsbildern verwiesen [1]. 


\section{Interessenkonflikt}

Der Autor erhielt in den vergangenen 3 Jahren Referentenund Beraterhonorare von den Unternehmen Alexion und Temmler.

Autorinnen/Autoren

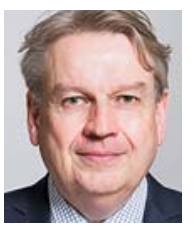

\section{Jörn Peter Sieb}

Jahrgang 1961, Prof. Dr. med., Facharzt für Neurologie, Geriatrie. Seit 2003 Chefarzt der Neurologischen Klinik, HELIOS Hanseklinikum Stralsund, seit 10/2019 zusätzlich auch Chefarzt der dortigen Klinik für Geriatrie und Frührehabilitation. Einer seiner klinischen und wissenschaftlichen Arbeitsschwerpunkte sind die myasthenen Syndrome mit einer Betreuung von über 300 Myastheniepatienten.

\section{Korrespondenzadresse}

Prof. Dr. med. Jörn Peter Sieb

Neurologische Klinik

HELIOS Hanseklinikum Stralsund

Große Parower Straße 47-53

18435 Stralsund

E-Mail: joern-peter.sieb@helios-gesundheit.de
Zitierweise für diesen Artikel

Bischoff C, Buchner H, Hrsg. SOPs Neurophysiologische Diagnostik, 1. Auflage. Stuttgart: Thieme 2018: 56-60.

Literatur

[1] Engel AG, Shen XM, Selcen D et al. Congenital myasthenic syndromes: pathogenesis, diagnosis, and treatment. Lancet Neurol 2015; 14: 420-434

[2] Guptill JT, Sanders DB, Evoli A. Anti-MuSK antibody myasthenia gravis: clinical findings and response to treatment in two large cohorts. Muscle Nerve 2011; 44: 36-40

[3] Sanders DB, Cao L, Massey JM et al. Is the decremental pattern in Lambert-Eaton syndrome different from that in myasthenia gravis? Clin Neurophysiol 2014; 125: 1274-1277

[4] Sieb JP. Myasthenia gravis: an update for the clinician. Clin Exp Immunol 2014; 175: 408-418

[5] Titulaer M], Lang B, Verschuuren J]. Lambert-Eaton myasthenic syndrome: from clinical characteristics to therapeutic strategies. Lancet Neurol 2011; 10: 1098-1107

\section{Bibliografie}

DOI https://doi.org/10.1055/a-1022-2375

Neurologie up2date 2020; 3: 233-238

(c) Georg Thieme Verlag KG Stuttgart · New York

ISSN 2511-3453 